\title{
Respostas termorregulatórias de crianças no exercício em ambiente de calor
}

\author{
Thermoregulatory responses of children exercising in a hot environment
}

\author{
Luis Henrique L. S. Gomes ${ }^{1}$, Miguel Araújo Carneiro-Júnior ${ }^{2}$, João Carlos B. Marins ${ }^{3}$
}

\section{RESUMO}

Objetivo: Revisar as possíveis peculiaridades nos mecanismos biológicos referentes às respostas termorregulatórias e sudorípara específicas no exercício realizado por crianças em ambiente de calor.

Fontes de dados: Foi feita uma revisão de 47 artigos publicados entre 1960 e 2011 nas bases de dados eletrônicos MedLine e SciELO Brasil, com a utilização dos seguintes descritores: 'crianças', 'calor', 'sudorese', 'termorregulação', 'glândula sudorípara' e 'exercício', sendo usados isoladamente ou em combinação, além de uma tese de doutorado sobre o assunto.

Síntese dos dados: Em pré-púberes, a taxa de sudorese durante o esforço é menor em comparação aos adultos. Crianças possuem características termorregulatórias diferenciadas, apresentando um débito de suor por glândula muito menor. A maior razão entre área de superfície e massa corporal faz com que crianças absorvam mais calor durante o exercício sob estresse térmico, elevando o risco de apresentarem sintomas de hipertermia. O maior fluxo sanguíneo para a pele contribui com um melhor controle da homeostase térmica de crianças. $\mathrm{O}$ menor tamanho da glândula, a menor sensibilidade colinérgica, os níveis baixos de catecolaminas circulantes durante o esforço e a falta de hormônio androgênico explicam a ocorrência da baixa eliminação de suor no exercício realizado por crianças.

Conclusões: Crianças exibem glândulas sudoríparas imaturas. Assim, a prática de atividade física combinada a altas temperaturas não é bem tolerada havendo maior vulnerabilidade às lesões térmicas. No calor, deve-se ter um con- trole rigoroso da ingestão de líquidos e um monitoramento atencioso das condições climáticas para maior segurança na prática de exercícios.

Palavras-chave: crianças; exercício; sudorese; termorregulação; hipertermia induzida.

\section{ABSTRACT}

Objective: To review possible peculiarities in biological mechanisms related to responses of thermoregulatory and specific sweat glands in exercise performed by children in hot environments.

Data sources: Review of 47 articles published between 1960 and 2011 in the electronic databases MedLine and SciELO Brazil using the following key-words: 'children', 'heat', 'sweating', 'thermoregulation', 'sweat gland', and 'exercise', alone or in combination, in addition to a doctoral thesis about the subject.

Data synthesis: Pre-pubertal sweat rate during exercise is lower than among adults. Children have different thermoregulatory characteristics, with a small sweat output rate due to small sweat glands. High ratio between surface and body mass increases the absorption of heat during exercise under thermal stress in children, raising the risk of hyperthermia symptoms. However, great blood flow to skin contributes to the better control of thermal homeostasis in children. Small size of the gland, low cholinergic sensibility, low levels of circulating catecholamines during stress, and lack of androgenic hormone explain the occurrence of low elimination of sweat in exercises performed by children.
Instituição: Universidade Federal de Viçosa (UFV), Viçosa, MG, Brasil 'Pós-graduado em Futebol pela UFV, Viçosa, MG, Brasil

2Doutorando em Ciências Fisiológicas da Universidade Federal do Espírito Santo (Ufes), Vitória, ES, Brasil

${ }^{3}$ Professor Doutor do Departamento de Educação Física da UFV, Viçosa, MG, Brasil
Endereço para correspondência:

Luis Henrique L. S. Gomes

Rua Tiradentes, 153, apto 103 - João Bras

CEP 36570-000 - Viçosa/MG

E-mail: Isilame@gmail.com

Conflito de interesse: nada a declarar

Recebido em: 1/12/2011

Aprovado em: 4/4/2012 
Conclusions: Children present immature sweat glands. Thus, physical activity combined with high temperatures is not well-tolerated by children and youngsters, with great vulnerability to thermal injury. In the heat, strict control of fluid intake and attentive monitoring of weather conditions should have especial attention for the safe practice of exercises.

Key-words: children; exercise; body temperature regulation; hyperthermia, induced.

\section{Introdução}

A característica mais marcante da termorregulação de crianças que as diferencia dos adultos é sua menor produção de suor ${ }^{(1)}$. Em pré-púberes, a taxa de sudorese durante o esforço raramente excede $400 \mathrm{~mL} \cdot \mathrm{h}^{-1(2)}$. Outros estudos ${ }^{(3-7)}$ confirmam a reduzida produção de suor, o que diminui a capacidade de perda de calor da criança pelo mecanismo da evaporação, predispondo a um maior risco de lesões térmi$\operatorname{cas}^{(8)}$. Assim, sugere-se que a baixa produção de suor esteja relacionada à imaturidade da glândula sudorípara ${ }^{(9,10)}$.

Crianças possuem características fisiológicas e anatômicas diversas das dos adultos, com valores distintos de composição corporal, água e densidade óssea. Morfologicamente, este grupo apresenta maior razão da área de superfície e massa corporal, o que leva a um aumento mais rápido da temperatura do corpo quando existe estresse térmico pelo calor ${ }^{(1,10,11)}$.

Diferenças no sistema circulatório entre crianças e adultos também podem influenciar a resposta termolítica. Crianças apresentam menor volume cardíaco, plasmático e débito cardíaco para uma mesma carga de trabalho em comparação aos adultos ${ }^{(1)}$. Um menor débito cardíaco, neste caso, significa que menos sangue será resfriado pelo corpo, com prejuízo termorregulatório. Entretanto, quando as crianças se exercitam no calor, desviam grande parte do débito cardíaco para a pele na tentativa de melhor controlar a homeostase térmica ${ }^{(9,10)}$.

Existem poucos dados estatísticos sobre acidentes termorregulatórios com crianças e adolescentes. No entanto, em um torneio de futebol infantojuvenil nos Estados Unidos, realizado em clima quente, 34 jogadores foram hospitalizados apresentando sintomas de hipertermia ${ }^{(12)}$. Acidentes temorregulatórios podem ser evitados respeitando-se os horários mais adequados para a prática de exercícios e oferecendo boa quantidade de líquidos. No Brasil, é frequente observar que competições de futebol de base são realizadas em horários centrais do dia, expondo crianças ou jovens adolescentes a condições termorreguladoras críticas.
Entender os fenômenos biológicos referentes ao controle da temperatura corporal e à real necessidade hídrica de crianças torna-se extremamente importante para a fisiologia do exercício pediátrico, já que se observa a entrada cada vez mais precoce deste grupo no cenário esportivo. A busca por informações nesta área do conhecimento orienta estratégias de hidratação no esporte, principalmente de jovens atletas que se exercitam sob estresse térmico, além de promover maior segurança na prática de exercícios de crianças e adolescentes.

O objetivo deste estudo foi revisar as possíveis peculiaridades nos mecanismos biológicos referentes às respostas termorregulatórias e sudorípara específicas no exercício realizado por crianças em ambiente de calor.

\section{Fontes de Dados}

Foram selecionados artigos publicados em revistas indexadas entre 1960 e 2011, nos idiomas inglês e português, em bancos de dados eletrônicos (Medline e SciELO Brasil e Google Acadêmico), com utilização combinada ou isolada dos seguintes descritores: crianças, calor, sudorese, termorregulação, glândula sudorípara e exercício.

Dessa forma, o texto foi estruturado em dois tópicos. $\mathrm{O}$ primeiro refere-se ao controle da homeostase térmica no exercício realizado por crianças em ambiente quente, ressaltando as principais diferenças em relação aos adultos. Já o segundo tópico aborda a fisiologia da glândula sudorípara infantil com foco na baixa sudorese apresentada.

\section{Controle da homeostase térmica no exercício realizado por crianças em ambiente quente}

A perda de calor evaporativo é crucial para a manutenção da homeostase térmica, independentemente da idade. Em crianças, certas características fisiológicas, anatômicas e metabólicas específicas proporcionam respostas diferenciadas em relação ao exercício. Tradicionalmente, atribui-se a tal grupo menor eficiência em relação à perda de calor ${ }^{(13)}$. Essa ideia é confrontada por estudos que consideram a termorregulação das crianças tão eficiente quanto a dos adultos ${ }^{(1,6,7,10)}$, apesar da menor produção de suor.

O que foi observado, no entanto, é que a prática de atividade física combinada a altas temperaturas não é bem tolerada pelo grupo infantojuvenil, com maior vulnerabilidade às lesões térmicas ${ }^{(8)}$. Neste sentido, as estratégias de hidratação para crianças e adolescentes devem ser pautadas pelas principais peculiaridades da termorregulação de jovens 
atletas, sendo recomendado evitar atividades nos horários mais quente do dia.

A termorregulação é realizada por um sistema de controle fisiológico que consiste em: termorreceptores centrais e periféricos, sistema de condução aferente, controle central de integração dos impulsos térmicos e sistema de respostas eferentes, levando a respostas compensatórias ${ }^{(14)}$. Este sistema regula o equilíbrio entre produção (termogênese) e dissipação (termólise) do calor para manter a temperatura em aproximadamente $36,5^{\circ} \mathrm{C}$.

No hipotálamo, situa-se o sistema de controle central que regula a temperatura do corpo ao integrar os impulsos térmicos provenientes dos tecidos do organismo. Quando o impulso integrado excede ou fica abaixo da faixa limiar de temperatura, ocorrem respostas termorreguladoras autonômicas na tentativa de manter a temperatura do corpo adequada ${ }^{(14)}$.

Como observado, o exercício associado ao estresse térmico pelo calor impõe um grande desafio à homeostase térmica de crianças, podendo comprometer não só o desempenho, como também seu estado de saúde.

Embora os mecanismos físicos de perda de calor sejam semelhantes aos dos adultos, as taxas de termogênese e termólise diferem. Os humanos perdem calor por condução, convecção, radiação e evaporação. A evaporação do suor é a mais importante e dependente da produção de suor. Contudo, em pré-púberes este mecanismo encontra-se imaturo ${ }^{(7,9)}$. Além da menor produção de suor, crianças experimentam aumento mais rápido da temperatura central durante o exercício no calor, o que pode gerar um quadro de hipertermia ${ }^{(1,11,15)}$.

Outras características peculiares referentes à termorregulação em crianças devem ser ressaltadas. O calor metabólico da locomoção é superior em crianças, pois estas precisam de mais energia em comparação aos adultos em atividades como caminhada ou corrida ${ }^{(16,17)}$. Desta forma, as necessidades energéticas na criança podem ser até $30 \%$ mais elevadas do que nos adultos, o que pode ser atribuído à menor eficiência mecânica. Assim, o ganho de calor metabólico referente ao trabalho muscular aumenta mais em crianças.

Há outro fator importante em relação ao baixo metabolismo glicolítico no exercício moderado realizado por crianças. Menores níveis das enzimas lactato desidrogenase e fosfofrutoquinase limitam a velocidade da glicólise ${ }^{(18,19)}$. Dessa forma, a menor liberação de catecolaminas, decorrente do baixo metabolismo anaeróbio no exercício, limita a sudorese da criança, visto que os níveis de catecolaminas circulantes aumentam a sudorese por estimulação de betarreceptores adrenérgicos ${ }^{(20,21)}$.
Durante o esforço, jovens atletas apresentam menor débito cardíaco comparados aos adultos, desviando grande parte deste para a pele em condições de alta temperatura ${ }^{(10,17)}$, diminuindo a circulação central e evidenciando maior trabalho cardiovascular ${ }^{(22)}$. Sendo assim, pré-púberes respondem melhor pelo esfriamento por convecção e radiação do que pelo mecanismo de evaporação ${ }^{(23,24)}$. Isto ocorre, sobretudo, devido à maior vasodilatação cutânea e fluxo sanguíneo aumentado para a pele ${ }^{(10,25)}$, tornando possível o controle da temperatura. As crianças exibem uma superfície externa relativamente grande por unidade de peso corporal, o que faz com que seus corpos absorvam calor mais rapidamente em ambientes onde a temperatura ambiental ultrapassa à da pele.

A temperatura da pele é maior se comparada com a dos adultos submetidos ao esforço sob as mesmas condições ambientais ${ }^{(26)}$. A maior elevação da temperatura da pele é observada provavelmente devido ao aumento do fluxo sanguíneo e à vasodilatação cutânea ${ }^{(10,27)}$. No entanto, a perda de calor pela vasodilatação cutânea não pode compensar a função sudorípara subdesenvolvida e, consequentemente, uma elevação rápida da temperatura central é verificada ${ }^{(10)}$.

A evaporação do suor é a principal forma de dissipação do calor em humanos. Em condições de clima quente, uma menor produção deste fluido contribui para o aquecimento mais rápido do organismo, levando à menor tolerância ao exercício. No entanto, Inbar $e t a^{(7)}$ afirmam ser superior a perda de calor por evaporação quando corrigido pela massa corporal, representando maior eficiência da sudorese (evaporação/sudorese total), o que possibilita um menor acúmulo de calor em crianças durante o exercício.

Diante da controvérsia, aconselha-se controlar rigorosamente as condições de temperatura e umidade relativa do ar do local onde será realizado o exercício, principalmente nas estações mais quentes do ano, com o intuito de prevenir os sintomas indesejados da hipertermia. A Figura 1 apresenta um guia climático elaborado pela American Academy Pediatrics (AAP) para prevenir lesões térmicas causadas pelo calor em crianças ativas durante exercícios prolongados.

\section{Mecanismo sudoríparo infantil subdesenvolvido}

O sistema termorregulatório de pré-púberes é distinto por estar em desenvolvimento, podendo influenciar a capacidade do corpo de dissipar e se proteger do calor ${ }^{(7,28)}$. Para um melhor entendimento do mecanismo sudoríparo imaturo de crianças, é necessário compreender a fisiologia da glândula sudorípara, em especial nos eventos que desencadeiam a produção do suor. 


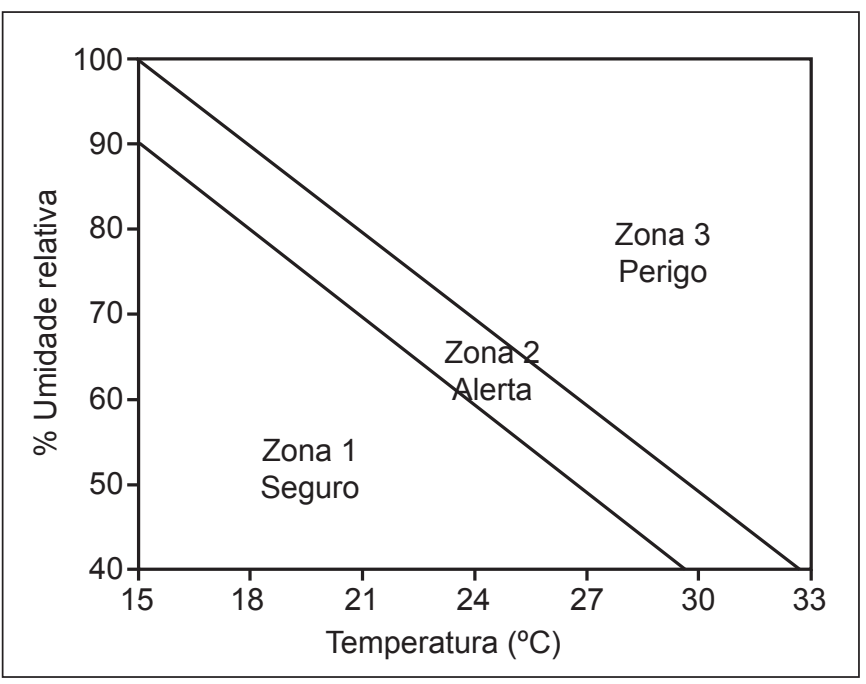

Figura 1 - Guia climático com divisão em três zonas de segurança: 1 (seguro); 2 (alerta) e 3 (perigo), adaptado da Academia Americana de Pediatria

Sinais eferentes oriundos da área pré-ótica do hipotálamo via tegumentar e região medular da rafe descem até o ramo ventral na medula espinhal. Este, por sua vez, comunica-se com o gânglio simpático por intermédio da fibra pré-ganglionar. A fibra simpática pós-ganglionar não mielinizada passa por meio da comunicação com um ramo cinzento, dando origem ao nervo sudomotor, o qual realiza sinapses diretamente nas glândulas sudoríparas e nos tecidos da pele ${ }^{(27)}$. Apesar da inervação simpática, o sistema responsável pela sudorese é o simpático colinérgico, sendo a acetilcolina a principal substância neurotransmissora envolvida na expulsão do suor, exibindo numerosos terminais ${ }^{(27)}$.

Quando a acetilcolina liga-se aos receptores muscarínicos da glândula, aumenta a concentração intracelular de íons cálcio. A concentração de cálcio elevada desencadeia o aumento da permeabilidade de $\mathrm{K}^{+}$e $\mathrm{Cl}^{-}$, iniciando, assim, a liberação do líquido isotônico precursor na sua porção glandular ${ }^{(27)}$. O suor é formado pela secreção ativa de $\mathrm{Na}^{+}$e $\mathrm{Cl}^{-}$, com uma difusão passiva de água pela membrana. Ao passar pelo ducto, indo até o poro, ocorre a diminuição da concentração de constituintes do fluido, principalmente $\mathrm{Na}^{+} \mathrm{e} \mathrm{Cl}^{-}$, por meio da troca de $\mathrm{K}^{+}$na membrana basolateral. Tal mecanismo é regulado pela bomba sódio-potássio ATPase ${ }^{(29)}$.

O suor é hipotônico em relação ao plasma sanguíneo. Em crianças e adolescentes é ainda mais hipotônico, já que perdem menos eletrólitos na transpiração ${ }^{(5,28)}$. Isso reduz o risco de um quadro de hiponatremia ou hipocalemia em crianças em condição de exercício.

A liberação do suor ocorre de forma pulsátil. Portanto, o mecanismo de expulsão do fluido é regulado pela rápida hidrólise da acetilcolina pela acetilcolinesterase ${ }^{(30)}$. A acetilcolina induz diretamente a atividade sudomotora central por meio da ativação dos receptores muscarínicos e, indiretamente, dos receptores nicotínicos oriunda da atividade axônica reflexa ${ }^{(31)}$.

Em humanos, o número de glândulas sudoríparas varia entre 1,6 e 4 milhões distribuídas por toda superfície corporal, existindo correlação positiva entre o número de glândulas e a capacidade de sudorese ${ }^{(30)}$. Após dois e três anos de idade, não há formação de novas glândulas ${ }^{(30)}$.

Alterações na taxa de sudorese podem resultar em mudança da densidade das glândulas ativadas pelo calor e/ou pelo débito de suor por glândula durante cada expulsão ${ }^{(26,30)}$. O efeito, tanto do crescimento e da maturação como da aclimatação ao calor, aumenta o estímulo colinérgico da glândula sudorípara. Além disso, ocorre concomitantemente à aceleração dos estímulos direcionados ao sistema nervoso simpático periférico ${ }^{(31)}$.

Com o crescimento físico, em especial da área de superfície corporal, observa-se a diminuição da densidade de glândulas ativadas pelo calor. Esse fenômeno não favorece o aumento da transpiração, mas, em compensação, a glândula aumenta de tamanho (hipertrofia), elevando a quantidade de suor eliminado ${ }^{(9,10)}$. O crescimento e a maturação propiciam o aumento da habilidade das glândulas, contudo, de forma não uniforme. Isto significa que algumas áreas podem apresentar maior sensibilidade colinérgica em relação a outras ${ }^{(10)}$.

$\mathrm{O}$ aumento na taxa de sudorese acontece por três fatores: aumento do número de glândulas ativadas pelo calor; aumento de suor por glândula; e combinação das duas formas ${ }^{(30,32)}$. Por outro lado, a desidratação inibe a sudorese primariamente por meio de mecanismos centrais, devido ao aumento da osmolalidade do sangue ${ }^{(30)}$, o que diminui a sensibilidade da glândula e compromete a capacidade de controle da temperatura ${ }^{(33)}$. Sabe-se que a desidratação superior a $2 \%$ do peso corporal prejudica o rendimento em adultos, contudo, em crianças, apenas $1 \%$ já compromete o exercício, além de aumentar o risco de um quadro de hipertermia ${ }^{(34)}$.

O tamanho menor da glândula sudorípara de crianças explica em grande parte sua produção de suor, pois, quanto menor a glândula, menor sua porção secretória ${ }^{(10)}$. Shibasaki et al ${ }^{(30)}$ afirmam haver correlação positiva entre tamanho individual da glândula e taxa de sudorese. Corroborando este estudo, Falk et al ${ }^{(35)}$ afirmam que o tamanho da glândula sudorípara pode estar relacionado com a idade e a altura do indivíduo. Com o crescimento e a maturação, a hipertrofia da glândula 
sudorípara associa-se ao aumento dos estímulos colinérgicos. Portanto, as respostas periféricas das glândulas sudoríparas são reguladas principalmente por estímulos colinérgicos, embora também possam responder aos adrenérgicos ${ }^{(36)}$.

$\mathrm{O}$ treinamento físico e a aclimatação podem modificar a sudorese. Um bom condicionamento físico propicia maior controle periférico, ocorrendo aumento da glândula (hipertrofia), mesmo em pré-púberes. Consequentemente, isto induz à maior sudorese ${ }^{(37)}$.

Apesar de apresentar maior densidade das glândulas ativadas pelo calor devido ao pequeno tamanho, a criança mostra débito de suor por glândula muito inferior em relação aos adultos ${ }^{(11)}$. Sobre esta questão, Sato e Sato ${ }^{(38)}$ relataram indivíduos adultos com precariedade da função sudorípara, apresentando menor tamanho da glândula (hipotrofia) e expulsão de suor por glândula. Assim, estudos envolvendo crianças sugerem que a sensibilidade colinérgica explica o baixo volume de suor ${ }^{(9,10)}$.

A ação de hormônios androgênios também pode modular o mecanismo de controle da sudorese. O aumento da transpiração, que ocorre durante a maturação, pode estar relacionado à maior liberação de hormônios masculinizantes ${ }^{(1,10)}$. Kawahata $^{(3)}$, em 1960, por meio de injeções farmacológicas, identificou que a testosterona aumentava a resposta da sudorese, enquanto o estradiol a inibia, sugerindo uma possível diferença sexual.

Estudo de Araki et a ${ }^{(39)}$ mostrou progresso dramático da sudorese após a puberdade. Entretanto, a diversidade sexual em crianças não foi verificada, atribuindo a falta dos efeitos da testosterona como responsável pela baixa eliminação do suor de pré-púberes de ambos os sexos. Parece não haver diferenças na taxa de sudorese entre meninos e meninas quando os mesmos apresentam níveis similares de atividade física e não são aclimatados ${ }^{(5)}$.

$\mathrm{Na}$ mesma direção, Inoue et $a l^{(10)}$ referem-se à falta de efeitos de certos hormônios masculinos androgênicos como uma das causas da produção diminuída de suor da criança. No entanto, o papel da testosterona na regulação de suor não está firmemente consolidado. Com a chegada e o avançar do período puberal, identificou-se em vários estudos o aumento da taxa de sudorese ${ }^{(5,9,34)}$. Falk et al ${ }^{(40)}$ encontraram um menor metabolismo glicolítico da glândula sudorípara infantil, sugerindo relacionar-se com a baixa sudorese apresentada.

A liberação de catecolaminas pelas adrenais também pode modular a sudorese. $\mathrm{O}$ aumento de tais hormônios na circulação, principalmente a adrenalina, estimula a sudorese pela ativação de betarreceptores da glândula sudorípara ${ }^{(20,21)}$.
Considerando a ação do sistema autônomo simpático no esforço realizado por pré-púberes, baixos níveis de adrenalina circulante durante o esforço moderado indicam menor participação da glicólise anaeróbica. Com menos adrenalina liberada, diminui-se a ativação dos receptores adrenérgicos das glândulas ${ }^{(20)}$. Quanto a esta questão, Tian $e t a l^{(21)}$ afirmam que, embora as catecolaminas não sejam determinantes para provocar a sudorese, são necessárias porque induzem maior responsividade secretória da glândula sudorípara. Em divergência, estudo recente $\mathrm{e}^{(41)}$ em modelo animal identificou que o bloqueio local dos betarreceptores adrenérgicos não afetou a sudorese durante o exercício.

Ao desenhar um planejamento de reposição hídrica para jovens engajados em treinamentos regulares e sistematizados, verificou-se a necessidade de quantificar individualmente a perda hídrica, principalmente pela sudorese no exercício $^{(42)}$. Jovens atletas devem ser orientados para que desenvolvam bons hábitos de hidrataçãa ${ }^{(34,43)}$. Todavia, ainda não existe consenso sobre a recomendação de hidratação a crianças e adolescentes fisicamente ativos ou jovens atle$\operatorname{tas}^{(44)}$. Estima-se que o balanço hídrico de crianças sedentárias seja de 1,6L por dia ${ }^{(45)}$. Dessa forma, crianças ativas devem ingerir um valor superior a este para assegurar a boa hidratação.

Apesar dos valores inferiores na taxa de sudorese apresentados em relação aos adultos, as estratégias de hidratação para este público não é menos importante, já que crianças não costumam repor os líquidos adequadamente ${ }^{(46-48)}$, podendo apresentar desidratação.

\section{Conclusões}

Crianças e adolescentes apresentam respostas termorregulatórias diferenciadas em relação ao exercício. No estresse térmico pelo calor, há aumento mais rápido da temperatura corporal, o que predispõe ao maior risco de lesões térmicas.

A evaporação do suor é a principal forma de dissipação do calor em humanos. No exercício realizado por crianças em ambiente quente, o valor da taxa de sudorese é menor, principalmente devido a mecanismos sudoríparos periféricos subdesenvolvidos. Portanto, existe um menor débito de suor por glândula, a qual, por sua vez, apresenta tamanho e sensibilidade colinérgica inferiores.

A liberação mais baixa de catecolaminas circulantes durante o esforço realizado por pré-púberes parece não 
favorecer grande eliminação do suor. A falta de hormônios masculinizantes foi atribuída como uma das causas para a menor sudorese em pré-púberes. No entanto, seu papel não está claro.

\section{Referências bibliográficas}

1. Rowland T. Thermoregulation during exercise in the heat in children: old concepts revisited. J Appl Physiol 2008;105:718-24.

2. American College of Sports Medicine, Sawka MN, Burke LM, Eichner ER, Maughan RJ, Montain SJ et al. American College of Sports Medicine position stand. Exercise and fluid replacement. Med Sci Sports Exerc 2007;39:377-90.

3. Kawahata A. Sex differences in sweating. In: Yoshimura H, Ogata K, Itoh S, editors. Essential problems in climatic physiology. Kyoto: Nankodo; 1960. p. 169-84.

4. Bar-Or O. Temperature regulation during exercise in children and adolescents. In: Lamb DR, Gisolfi CV. Perspectives in exercise science and sports medicine: youth, exercise, and sport. Indianapolis: Benchmark Press; 1989. p. 335-68.

5. Meyer F, Bar-Or O, MacDougall D, Heigenhauser GJ. Sweat electrolyte loss during exercise in the heat: effects of gender and maturation. Med Sci Sports Exerc 1992;24:776-81.

6. Shibasaki M, Inoue $\mathrm{Y}$, Kondo N, Iwata A. Thermoregulatory responses of prepubertal boys and young men during moderate exercise. Eur J Appl Physiol Occup Physiol 1997;75:212-8.

7. Inbar O, Morris N, Epstein Y, Gass G. Comparison of thermoregulatory responses to exercise in dry heat among prepubertal boys, young adults, and older males. Exp Physiol 2004;89:691-700.

8. American Academy Pediatrics; Committee on Sports Medicine and Fitness. Climatic heat stress and the exercising child and adolescent. Am Acad Ped 2000;106:158-9.

9. Shibasaki M, Inoue $\mathrm{Y}$, Kondo N. Mechanisms of underdeveloped sweating responses in prepubertal boys. Eur J Appl Physiol Occup Physiol 1997;76:340-5.

10. Inoue Y, Kuwahara T, Araki T. Maturation- and aging-related changes in the heat loss effector function. J Physiol Anthropol Appl Human Sci 2004;23:289-94.

11. Bar-Or O. Children's Responses to Exercise in Hot Climates: implications for performance and health. Sport Science Exchange [homepage on the Internet]. 1994;49 [cited 2009 March 26]. Available from: http://www.gssiweb. com/Article_Detail.aspx?articleid=22

12. Shirreffs SM, Aragon-Vargas LF, Chamorro M, Maughan RJ, Serratosa L, Zachwieja JJ. The sweating response of elite professional soccer players to trainning in the heat. Int J Sports Med 2005;26:90-5.

13. Bar-Or O, Wilk B. Water and electrolyte replenishment in the exercising child. Int J Sport Nutr 1996;6:93-9.

14. Romanovsky AA. Thermoregulation: some concepts have changed. Functional architecture of the thermoregulatory system. Am J Physiol Regul Integr Comp Physiol 2007;292:R37-46.

15. Rivera-Brown AM, Gutiérrez R, Gutiérrez JC, Frontera WR, Bar-Or O. Drink composition, voluntary drinking, and fluid balance in exercising, trained, heatacclimatized boys. J Appl Physiol 1999;86:78-84.

16. Bar-Or O. Nutrition for child and adolescent athletes. Sport Science Exchange [homepage on the Internet]. 2000;13 [cited 2009 May 19]. Available from: http:// cla.pointstreaksites.com/files/uploaded_documents/357/527_Nutrition_child_ adolescent.pdf

17. Falk B, Dotan R. Children's thermoregulation during exercise in the heat: a revisit. Appl Physiol Nutr Metab 2008;33:420-7.

18. Riddell MC. The endocrine response and substrate utilization during exercise in children and adolescents. J Appl Physiol 2008;105:725-33.

19. Kaczor JJ, Ziolkawski W, Popinigis J, Tarnopolsky MA. Anaerobic and aerobic enzyme activities in humam skeletal muscle from children and adults. Pediatr Res 2005;57:331-5.

20. Robertshaw D. Neuroendocrine control of sweat glands. J Invest Dermatol 1977;69:121-9.
As estratégias de hidratação no esporte para o público jovem sob influência de estresse térmico devem ser orientadas de acordo com as características distintas referentes à termorregulação e ao exercício.
21. Tian H, Habecker B, Guidry G, Gurtan A, Rios M, Roffler-Tarlov S et al Catecholamines are required for the acquisition of secretory responsiveness by sweat glands. J Neurosci 2000;20:7362-9.

22. Buchheit $M$, Duché $P$, Laursen $P B$, Ratel $S$. Postexercise heart rate recovery in children: relationship with power output, blood pH, and lactate. Appl Physiol Nutr Metab 2010;35:142-50.

23. Meyer F, Laitano O, Bar-Or O, McDougall D, Heigenhauser GJ. Effect of age and gender on sweat lactate and ammonia concentrations during exercise in the heat. Braz J Med Biol Res 2007;40:135-43.

24. Naughton GA, Carlson JS. Reducing the risk of heat-related decrements the physical activity in young people. J Sci Med Sport 2008;11:58-65.

25. Shibasaki M, Inoue Y, Kondo M, Aoki K, Hirata K. Relationship between skin blood flow and sweating rate in prepubertal boys and young men. Acta Physiol Scand 1999;167:105-10.

26. Davies CT. Thermal responses to exercise in children. Ergonomics 1981;24:55-61.

27. Shibasaki M, Crandall CG. Mechanisms and controllers of eccrinne sweating in humans. Front Biosci (Schol Ed) 2010;2:685-96.

28. Bar-Or O, Rowland T. Climate, body fluids, and the exercising child. In: Pediatric exercise medicine: from physiologic principles to health care application. Champagne: Human Kinetics; 2004. p. 69-101.

29. Quinton PM, Tormey JM. Localization Na/K- ATPase in the secretory and reabsortive epithelia of perfused eccrine sweat glands: a question to the role of the enzyme in secretion. J Membrane Biol 1976;29:383-99.

30. Shibasaki M, Wilson TE, Crandall CG. Neural control and mechanisms of eccrine sweating during heat stress and exercise. J Appl Physiol 2006;100:1692-701.

31. Lee JB, Kim TW, Shin YO, Min YK, Yang HM. Effect of the Heat-exposure on peripheral sudomotor activity including the density of active sweat glands and single sweat gland output. Korean J Physiol Pharmacol 2010;14:273-8.

32. Kondo N, Shibasaki M, Aoki K, Koga S, Inoue Y, Crandall CG. Function of human eccrine sweat glands during dynamic exercise and passive heat stress. J Appl Physiol 2001;90:1877-81.

33. Sawka MN, Montain SJ. Fluid and electrolyte supplementation for exercise heat stress. Am J Clin Nutr 2000;72 (Suppl 2):564S-72.

34. Rossi L, Reis VA, de Azevedo CO. Dehydration and rehydration recommendations for physically active children. Rev Paul Pediatr 2010;28:337-45.

35. Falk B, Bar-Or O, Calvert R, MacDougall JD. Sweat gland response to exercise in the heat among pre-, mid-, and late-pubertal boys. Med Sci Sports Exerc 1992;24:313-9.

36. Sato K. The physiology, pharmacology, and biochemistry of the eccrine sweat gland. Rev Physiol Biochem Pharmacol 1977;79:51-131.

37. Ichinose-Kuwahara T, Inoue $Y$, Iseki $Y$, Hara S, Ogura $Y$, Kondo N. Sex differences in the effects of physical training on sweat gland responses during a graded exercise. Exp Physiol 2010;95:1026-32.

38. Sato K, Sato F. Individual variations in structure and function of human eccrine sweat gland. Am J Physiol 1983;245:R203-8.

39. Araki T, Toda Y, Matsushita K, Tsujino A. Age differences in sweating during muscular exercise. J Physical Fitness Japan 1979;28:239-48.

40. Falk B, Bar-Or O, MacDougall JD, McGillis L, Calvert R, Meyer F. Sweat lactate in exercising children and adolescents of varying physical maturity. $J$ Appl Physiol 1991;71:1735-40.

41. Buono MJ, Tabor B, White A. Localized $\beta$-adrenergic receptor blockade does not affect sweating during exercise. Am J Physiol Regul Integr Comp Physiol 2011;300:R1148-51. 
42. Maughan RJ, Leiper JB, Shirreffs SM. Factors influencing the restoration of fluid and electrolyte balance after exercise in the heat. $\mathrm{Br} \mathrm{J}$ Sports Med 1997;31:175-82.

43. Gomes LH. Hydration care in young soccer players. Rev Bras Futebol 2010;3:38-47.

44. Juzwiak CR, Paschoal VC, Lopez FA. Nutrition and physical activity. J Pediatr (Rio J) 2000;76 (Suppl 3):S349-58.

45. Petrie HJ, Stover EA, Horswill CA. Nutritional concerns for the child and adolescent competitor. Nutrition 2004;20:620-31.
46. Santana JR, Rivera-Brown AM, Frontera WR, Rivera MA, Mayol PM, Bar-Or O. Effect of drink pattern and solar radiation on thermoregulation and fluid balance during exercise in chronically heat acclimatized children. Am J Human Biol 1995; 7:643-50.

47. Wilk $\mathrm{B}, \mathrm{Bar}-\mathrm{Or} \mathrm{O}$. Effect of drink flavor and $\mathrm{NaCl}$ on voluntary drinking and hydration in boys exercising in the heat. J Appl Physiol 1996;80:1112-7.

48. Perrone CA. Estado de hidratação, sudorese e reidratação durante uma sessão de treino no calor em jovens praticantes de diferentes esportes [tese de doutorado]. Porto Alegre (RS): UFRG; 2010. 